\title{
La ablación por láser como sistema de control analítico en metales con recubrimientos cerámicos
}

\author{
B. Ferrari ${ }^{(*)}$, R. Moreno ${ }^{(*)}$, T. Benito ${ }^{(* *)}$, M.T. Larrea ${ }^{(* *)}$ y J.C. Fariñas ${ }^{(*)}$
}

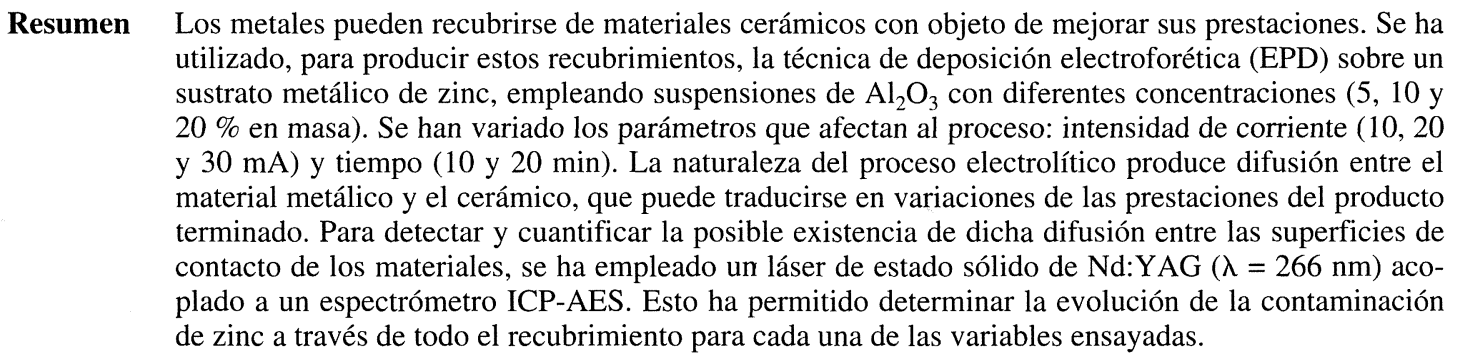

Palabras clave: Recubrimientos cerámicos sobre metales. Análisis químico. Ablación por láser. Espectrometría de ICP.

\section{Laser ablation as an analytical control system for ceramic coatings on metals}

\begin{abstract}
Metals can be coated with ceramic materials with the aim of improving their applications. Electrophoretic deposition (EPD) over a metallic substrate of zinc was used to produce these coatings, using $\mathrm{Al}_{2} \mathrm{O}_{3}$ slurries of several concentrations (5, 10 and $20 \%$ in mass). The parameters which control the process were modified: electric intensity $(10,20$ and $30 \mathrm{~mA})$ and time $(10$ and 20 $\mathrm{min})$. The nature of the electrolitic process determines a diffusion between the metallic and the ceramic materials, that may produce a variation of the properties of the final product. In order to detect and quantify the possible existence of that diffusion between the contact surfaces of both materials, a solid state laser of Nd:YAG $(\lambda=266 \mathrm{~nm})$ coupled to an ICP-AES spectrometer was used. This has allowed to determine the evolution of zinc contamination for each of the variables assayed through the whole coating.
\end{abstract}

Keywords: Ceramic coatings on metals. Chemical analysis. Laser ablation. ICP spectrometry.

\section{INTRODUCCIÓN}

La unión de materiales de características tan dispares como los metálicos y los cerámicos ofrece la posibilidad de obtener materiales compuestos con las propiedades eléctricas y mecánicas de los meta-

(*) Instituto de Cerámica y Vidrio (CSIC). Ctra. de Valencia, km 24,3. Arganda del Rey 28500-Madrid (España).

(**) Centro de Espectrometría Atómica. Fac. C.C. Geológicas. (U.C.M.). Ciudad Universitaria. 28040-Madrid (España).

(***) Centro Nacional de Investigaciones Metalúrgicas, CENIM (CSIC), Avda. de Gregorio del Amo, 8. 28040Madrid (España). les, protegidos en las condiciones más adversas de uso por una película delgada (en muchos casos, tan sólo de unos pocos micrómetros) de materiales cerámicos, lo que aumenta sus prestaciones iniciales.

La unión de un metal y una cerámica plantea diversos problemas, por lo que se ha desarrollado un gran número de técnicas para lograr estos recubrimientos (1). Entre ellas, cabe citar las de síntesis autopropagada, proyección de plasma, proyección de haces de iones, deposición química en fase vapor, deposición física en fase vapor, deposición por inmersión, deposición electroforética, etc. 
Todas ellas tienen limitaciones de procesamiento relacionadas con el rango de espesores que pueden obtenerse o el tipo de sustrato que puede recubrirse, y limitaciones prácticas como la alta tecnología necesaria en muchas de ellas, que hacen muy costoso su uso y lo restringe a aplicaciones concretas. En este sentido, la deposición electroforética (EPD) reúne gran cantidad de cualidades y posibles vías de procesamiento a partir de disoluciones (2) y suspensiones (3) o mediante vía sol-gel (4), entre otras. Todo ello hace que, en comparación con otras técnicas (5), la EPD sea una de las más utilizadas a la hora de procesar este tipo de materiales compuestos para su uso en distintas aplicaciones, tales como superconductores, semiconductores, dieléctricos, aislantes térmicos, etc. Esta técnica permite recubrir piezas metálicas de cualquier forma y espesor con películas cerámicas de diferente grosor. Sin embargo, debido a la propia naturaleza del proceso electrolítico, pueden existir problemas de difusión entre el material metálico y el cerámico, que pueden traducirse en una merma de las prestaciones finales del producto terminado. Resulta, por tanto, necesario poder detectar y cuantificar la posible existencia de dichas difusiones entre las superficies de contacto de los materiales.

Una de las técnicas más adecuadas para llevar a cabo este tipo de análisis es la ablación por láser (LA) acoplada a un espectrómetro de emisión atómica con fuente de plasma de acoplamiento inductivo (ICP-AES) (6). Esta técnica se basa en la irradiación, con un láser, de una muestra situada dentro de una cámara de ablación, el cual produce en el punto de impacto una fusión-vaporización-atomización-ionización del material. Este vapor atómicoiónico es arrastrado por una corriente de argón, con lo que solidifica en forma de partículas micrónicas y submicrónicas que son introducidas en el espectrómetro de plasma y analizadas. Este proceso de "ablación" origina en la zona de irradiación de la muestra un pequeño agujero ("cráter") cuya forma, tamaño y profundidad depende de un gran número de variables, como pueden ser: características de la muestra, tipo de láser, longitud de onda del mismo, energía del pulso, etc. La irradiación con pulsos sucesivos permite ir penetrando desde la superficie de la muestra hacia su interior, analizando simultáneamente por espectrometría de ICP los elementos de interés. Consecuentemente, se puede llevar a cabo el análisis químico de prácticamente cualquier material, aislante o conductor, directamente sobre la muestra sólida, sin ningún tipo de preparación o modificación de ésta, tanto en su superficie como en su interior $\mathrm{y}$, además, de forma totalmente localizada (7).

El objetivo del presente trabajo es demostrar la capacidad de la técnica de LA-ICP-AES para evaluar la difusión que puede tener lugar entre el recu- brimiento cerámico y el sustrato metálico, lo que puede permitir la optimización de las variables implicadas en el proceso de obtención del recubrimiento.

\section{PARTE EXPERIMENTAL}

Para la obtención de los recubrimientos de $\mathrm{Al}_{2} \mathrm{O}_{3}$ sobre cinc se han utilizado suspensiones acuosas de $\mathrm{Al}_{2} \mathrm{O}_{3}$ con un contenido en sólidos de 5 , 10 y $20 \%$ en masa. Los ensayos de deposición electroforética se han realizado en condiciones de intensidad de corriente constante de 10,20 y 30 $\mathrm{mA}$, con tiempos de deposición de 10 y $20 \mathrm{~min}$.

Las partículas de $\mathrm{Al}_{2} \mathrm{O}_{3}$ en suspensión se encuentran cargadas negativamente, de forma que al aplicar una determinada intensidad de corriente migran hacia el ánodo o electrodo de trabajo. En este estudio se ha utilizado como electrodo de trabajo una placa de cinc, el cual, al actuar como ánodo, durante el proceso se oxida a $\mathrm{Zn}^{2+}$.

Con el fin de evaluar la posible contaminación en el depósito de $\mathrm{Al}_{2} \mathrm{O}_{3}$ procedente del electrodo metálico, se han analizado por espectrometría de ICP con ablación por láser los depósitos obtenidos en diferentes condiciones de trabajo.

Como sistema de LA-ICP-AES, se ha empleado un equipo constituido por un láser de estado sólido de Nd:YAG Continuum, modelo Surelite, y una cámara de ablación fabricada en el laboratorio, un sistema Schneeberger de desplazamiento del portamuestras y un espectrómetro simultáneo de plasma Perkin Elmer, modelo Optima 3000 DV. La óptica (lentes de focalización y ventana de la cámara de ablación) está construida con un material de cuarzo transparente a la radiación láser (Suprasil).

De las cuatro longitudes de onda del láser de Nd:YAG (1064, 532, 355 y $266 \mathrm{~nm})$, en este trabajo se ha empleado exclusivamente la última de ellas, con unas condiciones de funcionamiento muy suaves $(1,11 \mathrm{kV}$ de tensión, $1 \mathrm{~Hz}$ de frecuencia, $9 \mathrm{~mW}$ de potencia y $9 \mathrm{~mJ}$ de energía por pulso). La determinación del cinc con el espectrómetro de ICP se ha efectuado utilizando su línea de emisión más intensa, situada a $213,856 \mathrm{~nm}(8)$.

Los depósitos se han irradiado con un número de pulsos láser suficiente para atravesarlos completamente, con el objeto de conocer la distribución del cinc a lo largo de todo el espesor de los mismos. La irradiación se ha efectuado, en primer lugar, sobre una de las superficies de cada material, para posteriormente realizar la irradiación sobre la otra superficie. Con ello, se tiene la seguridad de que se ha analizado correctamente todo el espesor del depósito o, al menos, las zonas que más interesan, que son las que van desde cada cara hacia el interior de la muestra. En todos los casos se han 
registrado las intensidades de emisión del cinc en función del número de pulsos, que es proporcional a la profundidad de penetración del láser. También se han controlado las emisiones del titanio y del carbono procedentes de la aleación de la que está fabricado el portamuestras, para determinar el instante en que el láser ha atravesado la muestra.

\section{RESULTADOS Y DISCUSIÓN}

La figura 1 recoge los perfiles de las intensidades de emisión del aluminio, carbono y titanio correspondientes a la irradiación de un depósito de $\mathrm{Al}_{2} \mathrm{O}_{3}$ de $1,5 \mathrm{~mm}$ de espesor, en el que se aprecia el instante en que la muestra es atravesada por la disminución de la intensidad del alumino y por el aumento de las intensidades del carbono y del titanio.

La figura 2 muestra los perfiles de intensidad de emisión del cinc en función del número de pulsos, correspondientes a la irradiación sobre la cara que estuvo en contacto con el electrodo de cinc, de las muestras obtenidas a distintas intensidades de corriente $(10,20$ y $30 \mathrm{~mA})$. Se observa que la presencia y distribución del cinc depende de la intensidad de corriente aplicada. Así, para las muestras depositadas con una intensidad de corriente de 10 $\mathrm{mA}$, se observa un pico estrecho, cuyo máximo

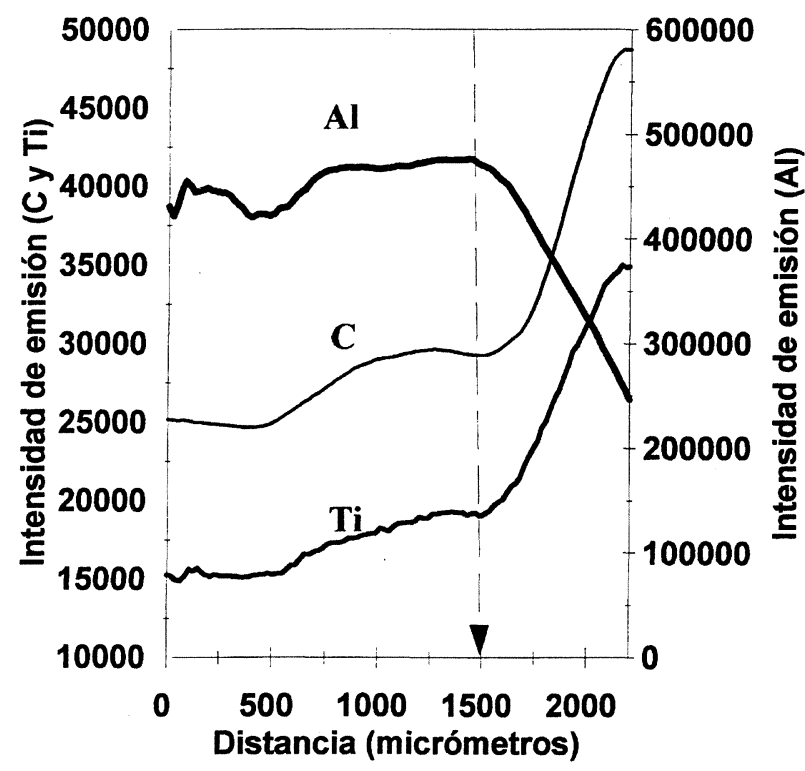

Fig. 1.- Perfiles de intensidades de emisión del aluminio, carbono y titanio obtenidos al atravesar completamente con el láser un depósito de $\mathrm{Al}_{2} \mathrm{O}_{3}$ de $1,5 \mathrm{~mm}$ de espesor.

FIG. 1.- Profiles of emission intensities of aluminium, carbon and titanium obtained to cross completely an $\mathrm{Al}_{2} \mathrm{O}_{3}$ deposit of $1.5 \mathrm{~mm}$ of thickness with the laser.

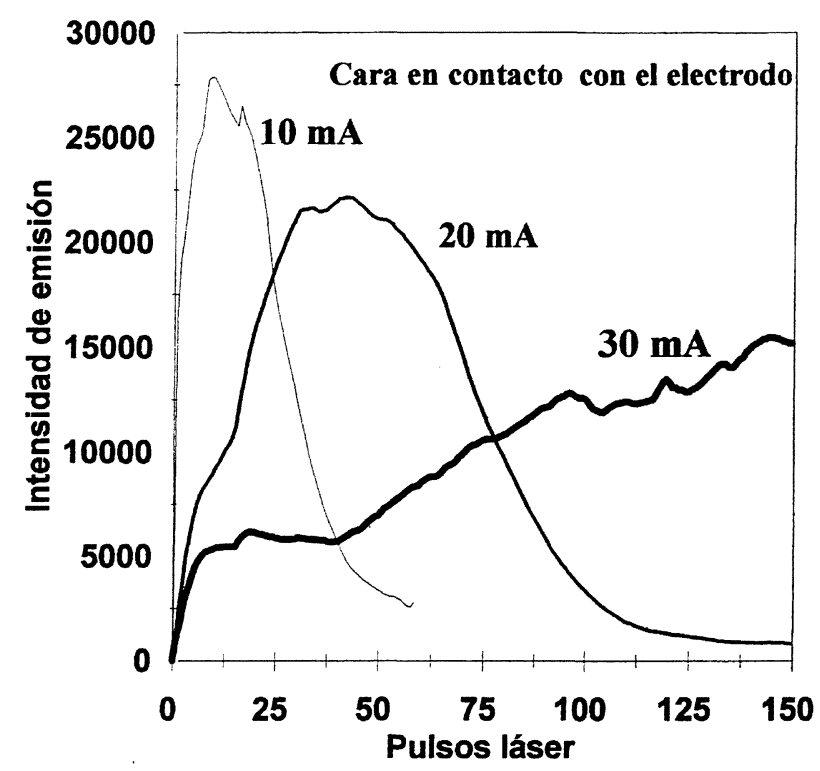

Fig. 2.- Perfiles de intensidades de emisión de cinc vs pulsos láser correspondientes a la irradiación de la superficie en contacto con el electrodo de los depósitos de $\mathrm{Al}_{2} \mathrm{O}_{3}$ obtenidos aplicando intensidades de corriente de 10, 20 y $30 \mathrm{~mA}$.

FIG. 2.- Profiles of emission intensities of zinc vs laser shots corresponding to the irradiation of the contact surface with the electrode of the $\mathrm{Al}_{2} \mathrm{O}_{3}$ deposits obtained by application of electrical intensities of 10, 20 and $30 \mathrm{~mA}$.

aparece al aplicar unos 15 pulsos láser. Para las muestras obtenidas a $20 \mathrm{~mA}$, el cinc presenta una señal menos intensa y más ancha, cuyo máximo aparece entre 30 y 40 pulsos láser, es decir, a una mayor profundidad que en el caso anterior. En las muestras obtenidas al aplicar una corriente de 30 $\mathrm{mA}$, no se observa un pico definido, sino que la intensidad de emisión del cinc aumenta progresivamente con el número de pulsos desde la superficie hasta el interior. Por otra parte, los perfiles de intensidad de emisión del cinc en función del número de pulsos correspondientes a las muestras irradiadas por la cara que permaneció en contacto con la suspensión durante el ensayo de EPD, se muestran en la figura 3. En este caso, las tres muestras presentan un máximo al comenzar la irradiación con el láser, siempre por debajo de 25 pulsos. Sin embargo, la intensidad del pico aumenta a medida que aumenta la intensidad de corriente aplicada para la formación del depósito, contrariamente a lo que sucede en la cara en contacto con el electrodo.

A partir de los perfiles de intensidad de emisión representados en las figuras 2 y 3 , se puede representar el perfil de la contaminación de cinc a lo largo del espesor de los depósitos de $\mathrm{Al}_{2} \mathrm{O}_{3}$, desde la cara en contacto con el electrodo hasta la opuesta, lo que se recoge en la figura 4. En la misma, se 


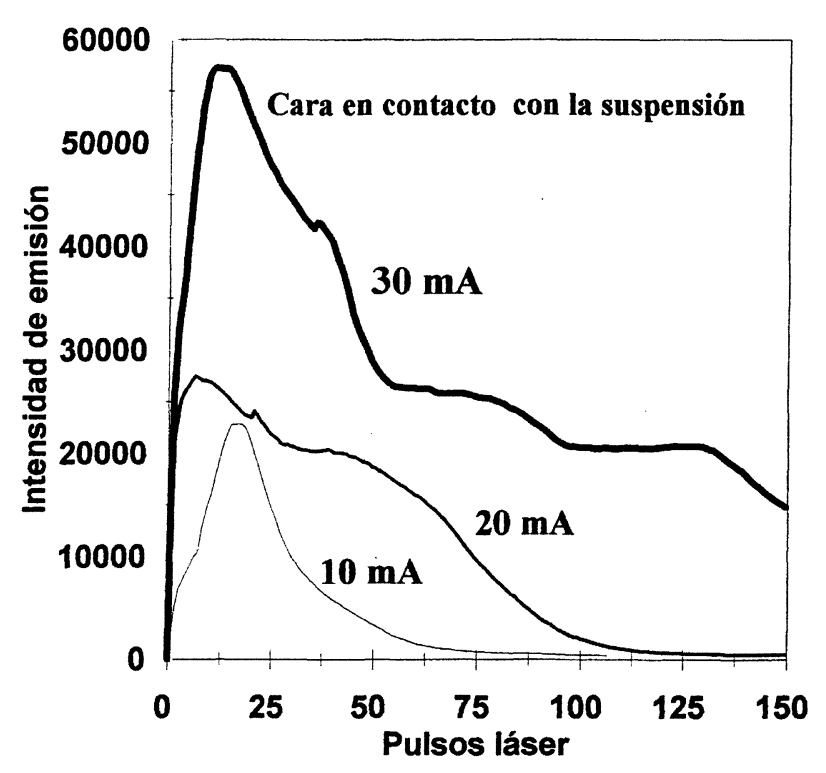

FIG. 3.- Perfiles de intensidades de emisión de cinc $v s$ pulsos láser correspondientes a la irradiación de la superficie en contacto con la suspensión de los depósitos de $\mathrm{Al}_{2} \mathrm{O}_{3}$ obtenidos aplicando intensidades de corriente de 10, 20 y $30 \mathrm{~mA}$.

FIG. 3.- Profiles of emission intensities of zinc vs laser shots corresponding to the irradiation of the contact surface with the slurry of the $\mathrm{Al}_{2} \mathrm{O}_{3}$ deposits obtained by application of electrical intensities of 10,20 and $30 \mathrm{~mA}$.

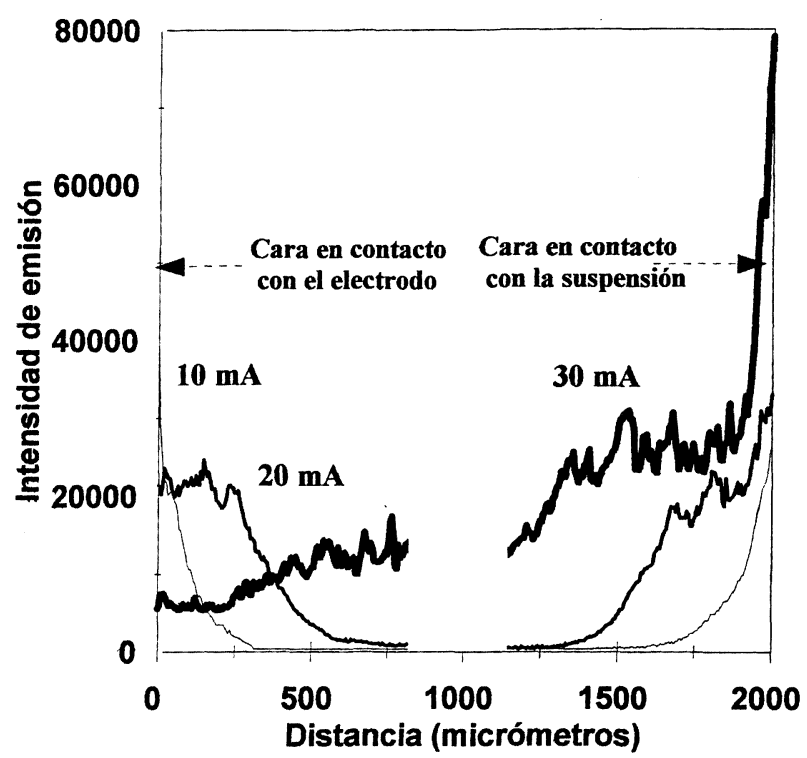

FIG. 4.- Evolución de la contaminación de cinc a lo largo del espesor de los depósitos de $\mathrm{Al}_{2} \mathrm{O}_{3}$ obtenidos aplicando intensidades de corriente de 10,20 y $30 \mathrm{~mA}$.

Fig. 4.- Evolution of the zinc contamination through the thickness of the $\mathrm{Al}_{2} \mathrm{O}_{3}$ deposits obtained by application of electrical intensities of 10,20 and $30 \mathrm{~mA}$. resume el comportamiento anteriormente mencionado. En la cara en contacto con el electrodo, la contaminación de cinc es tanto mayor cuanto menor es la intensidad de corriente, mientras que, por el contrario, en la superficie opuesta del depósito (en contacto con la suspensión) la contaminación de cinc aumenta a medida que lo hace la corriente aplicada. Es decir, cuando se aplican intensidades bajas, la contaminación es muy alta en la superficie de contacto con el electrodo, mientras que, para altas intensidades de corriente, se produce una mayor contaminación en la superficie opuesta. Esto sugiere la existencia de dos mecanismos distintos de contaminación, uno debido a la difusión de $\mathrm{Zn}^{2+}$ desde el electrodo a la suspensión, y otro simultáneo de migración de estos cationes hacia el cátodo de platino.

Este comportamiento es contrario al que cabría esperar, que sería simplemente una contaminación por formación y transporte de iones $\mathrm{Zn}^{2+}$ desde el electrodo hacia el depósito, concentrada en la cara del depósito que estuvo en contacto con el electrodo, y tanto mayor cuanto más alta fuera la intensidad de corriente aplicada en el proceso.

Las figuras 5 y 6 muestran los perfiles de la contaminación de cinc en función de la concentración de la suspensión utilizada para el recubrimiento y

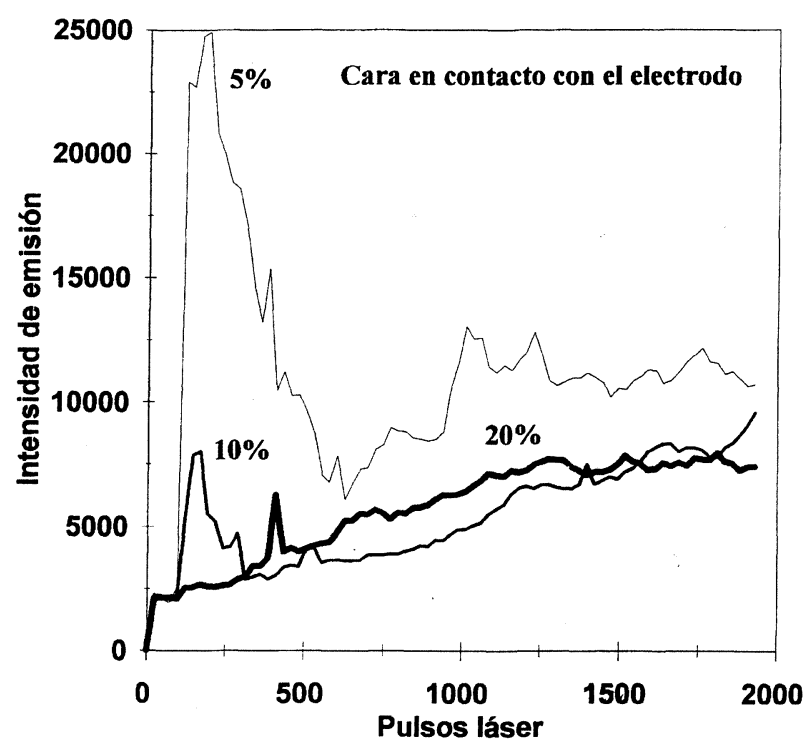

FIG. 5.- Perfiles de intensidades de emisión de cinc vs pulsos láser correspondientes a la irradiación de la superficie en contacto con el electrodo de los depósitos de $\mathrm{Al}_{2} \mathrm{O}_{3}$ obtenidos a partir de suspensiones con 5, 10 y $20 \%$ en masa.

FIG. 5.- Profiles of emission intensities of zinc vs laser shots corresponding to the irradiation of the contact surface with the electrode of the $\mathrm{Al}_{2} \mathrm{O}_{3}$ deposits obtained from slurries with 5, 10 and 20 mass \%. 


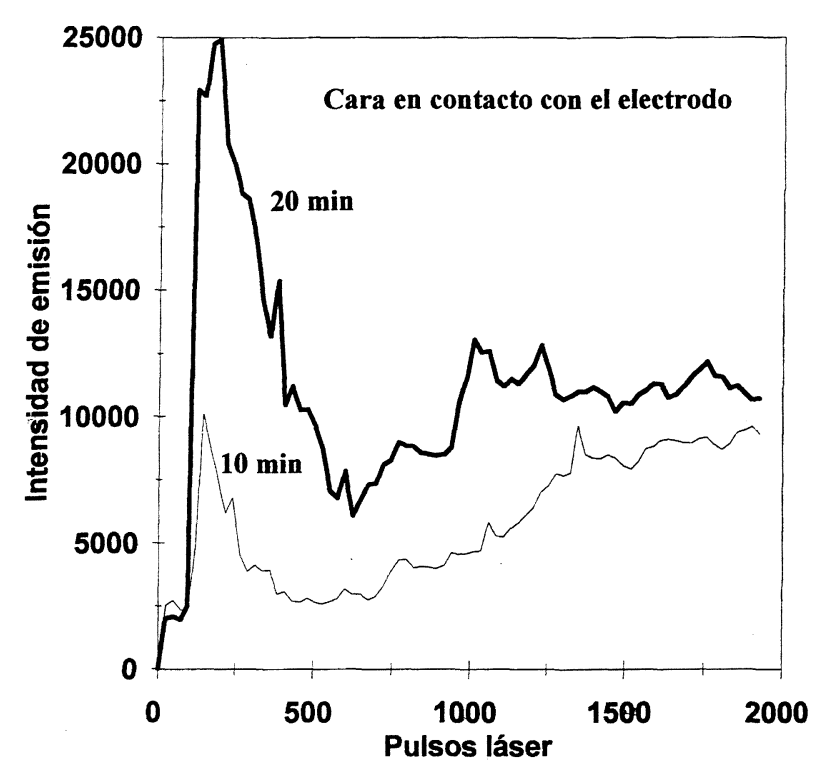

FIG. 6.- Perfiles de intensidades de emisión de cinc vs pulsos láser correspondientes a la irradiación de la superficie en contacto con el electrodo de los depósitos de $\mathrm{Al}_{2} \mathrm{O}_{3}$ obtenidos a 10 y $20 \mathrm{~min}$ de electrodeposición.

FIG. 6.- Profiles of emission intensities of zinc vs laser shots corresponding to the irradiation of the contact surface with the electrode of the $\mathrm{Al}_{2} \mathrm{O}_{3}$ deposits obtained at 10 and $20 \mathrm{~min}$ of electrodeposition.

de la duración del proceso de electrodeposición, respectivamente. En ellas, puede observarse cómo la difusión de cinc desde el sustrato metálico es tanto menor cuanto mayor es la concentración de la suspensión cerámica empleada y menor es la duración del proceso electrolítico.

\section{CONCLUSIONES}

La espectrometría de ICP con ablación por láser permite determinar la contaminación por difusión que se produce entre un sustrato metálico y su recubrimiento cerámico a través de todo su espesor. Esto posibilita la optimización de las variables que influyen en el proceso de fabricación y, consecuentemente, permite una mejora de las propiedades del nuevo material.

La aplicabilidad de la ablación por láser a cualquier tipo de material, unido a la capacidad de la espectrometría de ICP para determinar la mayoría de los elementos del sistema periódico, hace suponer el interés de esta nueva técnica en el estudio de difusiones metálicas existentes entre las capas de materiales con recubrimientos, independientemente de la naturaleza química de ambos.

\section{REFERENCIAS}

(1) Molera, P. Recubrimientos de Metales. MARCOMBO, S.A. Barcelona (España) 1989.

(2) Zhitomirsky, I., Gal-OR, L. y Klein, S. J. Mater. Sci. Lett., 14, 1995: 60-62.

(3) Mizuguchi, J., Sumi, K. y Muchi, T. J. Electrochem. Soc., 130, 1983: 1819-1825.

(4) Kishida, K., Tatsumisago, M. y Minami, T. J. Ceram. Soc. Jap., Int. Ed., 102, 1994: 336-340.

(5) Clark, D.E., Dalzell, W.J. y Folz, D.C. Ceram. Eng. Sci. Proc., 9, 1988: 1111-1118.

(6) PANG, H.M., Wiederin, D.R., Houk, R.S., Houk, E.S. y Yeung, E.S. Anal. Chem. 63, 1991: 390-394.

(7) Montaser, A. y Golightly, D.W. Inductively Coupled Plasmas in Analytical Atomic Spectrometry. VCH Publ. Inc. Nueva York (EE.UU.) 1992.

(8) Winge, R.K., Fassel, V.A., Peterson, V.J. y Floyd, M.A. Inductively Coupled Plasma-Atomic Emission Spectroscopy. Elsevier Science Publ. B.V. Amsterdam (Países Bajos ) 1985. 